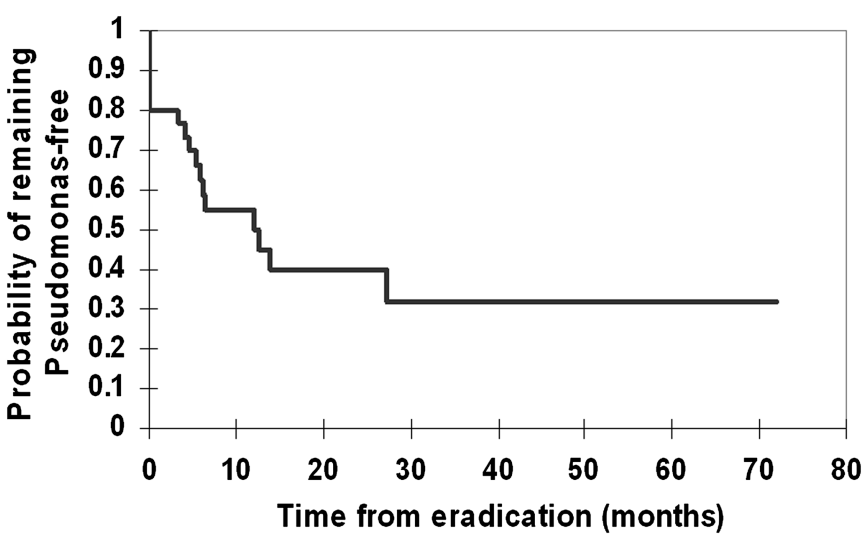

Abstract S130 Figure 1 Kaplan-Meier curve showing Pseudomonasfree time following eradication therapy.

eradication $(p=0.002)$. Mean number of hospital admissions per year were similar, at 0.39 pre-eradication and 0.29 post-eradication $(\mathrm{p}=\mathrm{NS})$. At first follow-up, 20 patients $(66.7 \%)$ reported overall clinical improvement, with reduced cough in 12 (40.0\%), reduced sputum volume in $14(46.7 \%)$ and reduced sputum purulence in $11(36.7 \%)$. Of the 21 patients followed up to 1 year, $6(28.6 \%)$ had further overall improvement and 13 (61.9\%) remained stable. Lung function was not affected by eradication therapy, with mean percentage predicted $\mathrm{FEV}_{1} 62.1 \%$ pre and $64.1 \%$ posteradication $(\mathrm{p}=\mathrm{NS})$.

Conclusion This study demonstrated that Pseudomonas eradication therapy can lead to prolonged clearance of this organism and highly significantly reduces exacerbation rate. This important outcome requires confirmation in a prospective study.

\section{S131 FATIGUE IN BRONCHIECTASIS: ITS RELATIONSHIP TO PSEUDOMONAS COLONISATION, DYSPNOEA AND AIRFLOW OBSTRUCTION}

doi:10.1136/thx.2010.150946.32

${ }^{1} \mathrm{~J} \mathrm{G}$ Macfarlane, ${ }^{1} \mathrm{H}$ Tedd, ${ }^{2} \mathrm{P}$ McAlinden, ${ }^{2} \mathrm{~T}$ Small, ${ }^{2} \mathrm{~L}$ Rostron, ${ }^{3} \mathrm{~J}$ Newton, ${ }^{4} \mathrm{~A}$ De Soyza. ${ }^{1}$ Freeman Hospital Respiratory Department, Newcastle upon Tyne, UK; ${ }^{2}$ William Leech Clinical Research Centre, Newcastle upon Tyne, UK; ${ }^{3}$ Institute of Ageing and Health, Newcastle University, Newcastle upon Tyne, UK; ${ }^{4}$ Institute of Cellular Medicine, Newcastle University, Newcastle upon Tyne, UK

Introduction and objectives Fatigue is a complex and disabling symptom in non-CF bronchiectasis (nCF-Br) and can be formally measured using the Fatigue Impact Scale (FIS). FIS scores of $>40$ out of 120 are clinically significant. The FIS score has been shown to be prognostic of premature death in Primary Biliary cirrhosis but is not linked to markers of organ dysfunction (liver function tests). As poorer outcomes have been recently reported in Pseudomonas infected $\mathrm{nCF}-\mathrm{Br}$ patients we aimed to measure the correlation between FIS scores and parameters of severity in nCF-Br, for example, Pseudomonas infection, degree of dyspnoea and airflow obstruction.

Methods $\mathrm{FEV}_{1} \%$ predicted, MMRC dyspnoea score (MMRCD) and FIS were recorded in stable adult nCF-Br patients attending specialist clinic. All previous sputum cultures isolating Pseudomonas aeruginosa were reviewed. Two groups of patients were studied: those with Pseudomonas 'colonisation' (organism cultured $\geq 2$ occasions, 3 months apart within 1-year period) and those with 'isolation' (organism cultured $\geq 1$ occasion). Statistical comparison used $\chi^{2}$, Fisher's correlation and Mann-Whitney $U$ tests.

Results 73F, 41M patients were included; average age 60 (range 24-90) with an average $\mathrm{FEV}_{1} 66 \%$ predicted (SD +/-26\%). 54
(47\%) patients had Pseudomonas isolation; 38 (33\%) patients had colonisation. Fatigue levels were similar in patients with and without colonisation (median 48.5 vs $36.5, p=0.31$ ). Significant fatigue (FIS $>40$ ) was more common in patients with Pseudomonas isolation (47\%) than those with no previous isolates ( $p=0.04$, $\mathrm{OR}=2.2$ ) However, fatigue levels, although increased, were not significantly different (median FIS 50 vs $32 ; p=0.064$ ). Fatigue correlated with MMRCD $(\mathrm{r}=0.54, \mathrm{p}<0.0001)$ but less well with $\mathrm{FEV}_{1} \%$ predicted $(\mathrm{r}=0.2, \mathrm{p}=0.04) . \mathrm{FEV}_{1} \%$ predicted was lower in patients with Pseudomonas colonisation (median $\mathrm{FEV}_{1} 49 \%$ vs $74 \%$, $\mathrm{p}=0.0007$ ) and in patients with Pseudomonas isolation (median $\mathrm{FEV}_{1}$ $52 \%$ vs $74 \%, p=0.002$ ).

Conclusions Pseudomonas infection (past or present) appears to be associated with greater clinically significant fatigue scores and poorer lung function. Fatigue doesn't strongly correlate with $\mathrm{FEV}_{1} \%$ predicted but is correlated with MMRCD. Further regression analysis of variables is underway to understand these inter relationships further. Systemic aspects of Pseudomonas infection may be different to other infections explaining the divergence.

\section{Clinical and translational observations in asthma}

\section{S132 AIRWAY DYSFUNCTION AND INFLAMMATION IN POOL AND NON-POOL BASED ELITE ENDURANCE ATHLETES}

doi:10.1136/thx.2010.150946.33

${ }^{1} \mathrm{~N}$ Martin, ${ }^{2} \mathrm{M}$ R Lindley, ${ }^{1} \mathrm{~B}$ Hargadon, ${ }^{1} \mathrm{~W}$ Monteiro, ${ }^{1} \mathrm{I} \mathrm{D}$ Pavord. ${ }^{1}$ Instiute for Lung Health, Glenfield Hospital, Leicester, UK; ${ }^{2}$ Department Human Sciences, Loughborough University, Loughborough, UK

Introduction Previous studies have suggested that eosinophilic airway inflammation is common in elite swimmers; the chemical pool environment often blamed. We set out to address this question in a cross-sectional study of 109 international athletes from a variety of sporting backgrounds.

Methods All had symptoms suggesting exercise-induced asthma and were either inhaled corticosteroid naïve or withdrew these for $>4$ weeks. $\beta_{2}$-agonists, exercise and caffeine were withheld for $8 \mathrm{~h}$ prior to testing. Symptoms were assessed using the Juniper ACQ, airways dysfunction using the eucapnic voluntary hyperventilation $(\mathrm{EVH})$ test and airways inflammation using exhaled nitric oxide $\left(\mathrm{FE}_{\mathrm{NO}}\right)$ and induced sputum eosinophil \% (eos). Athletes were classed as pool based if they exercised in an indoor pool environment for $>5 \mathrm{~h}$ per week, and non-pool based if they exercised in a pool for $<1 / 2$ h per week.

Results Demographic details were similar. Mean ( \pm SEM) \% fall in $\mathrm{FEV}_{1}$ post $\mathrm{EVH}$ was $18.96 \pm 1.701(\mathrm{n}=47)$ in pool and $11.39 \pm 1.249$ $(\mathrm{n}=62)$ in non-pool athletes (mean difference $7.569 ; 95 \%$ CI 3.480 to $11.66 ; p=0.0004), 76 \%$ of pool and $39 \%$ of non-pool athletes had a positive test ( $>10 \%$ fall). The geometric mean (log SD) eos (pool $2.667(0.797)$ ), non-pool 3.060 (0.867), $\mathrm{p}=0.802)$, and $\mathrm{FE}_{\mathrm{NO}}$ (pool 25.05 (1.570), non-pool 28.06 (1.475) ppb, p=0.914) was no different between groups; $14.9 \%$ of pool and $12.9 \%$ of non-pool athletes had eos $>3 \%$. \% fall in FEV1 had good correlation with $\log$ eos $(\mathrm{r}=0.551, \mathrm{p}<0.0001)$; a $25 \%$ fall being the optimum (AUC $0.89, \mathrm{p}<0.0001$, sens $79 \%$, spec 93\%). Log $\mathrm{FE}_{\mathrm{NO}}$ and $\log$ eos correlated strongly $(\mathrm{r}=0.644, \mathrm{p}<0.0001) ; \mathrm{FE}_{\mathrm{NO}}$ of $>47$ being most predictive of eos $>3 \%$ (AUC $0.912, p<0.0001$, sens $78 \%$, spec $92 \%$ ). Symptoms correlated poorly with either airways dysfunction or inflammation.

Conclusions Individual athletes with symptoms vary markedly in the levels of airways dysfunction and inflammation expressed. There is more airway dysfunction in pool athletes but not more eosinophilic airways inflammation suggesting that the pool 
environment if anything predisposes to airways dysfunction. Exhaled nitric oxide is a promising non-invasive means of assessing eosinophilic airways inflammation in all elite athletes.

\section{S133 EOSINOPHILIC AIRWAY INFLAMMATION IS ASSOCIATED WITH FEV1 DECLINE IN SEVERE ASTHMA}

doi:10.1136/thx.2010.150946.34

J Agbetile, D Desai, B Hargadon, P Bradding, A J Wardlaw, I D Pavord, R H Green, C E Brightling, S Siddiqui. Department of Infection, Immunity and Inflammation, Leicester University \& Institute for Lung Health, Glenfield Hospital, Leicester, UK

Background Severe asthma is a multidimensional disease, with recent evidence supporting the notion that eosinophilic airway inflammation (EAI) is an important driver for exacerbations. In addition EAI has been shown to be associated with airflow limitation in cross sectional studies. However, it remains to be established whether EAI may drive $\mathrm{FEV}_{1}$ decline.

Methods The severe asthma registry at Glenfield hospital, Leicester, was screened for patients with a physician diagnosis of asthma and at least 5 years of longitudinal data recording sputum eosinophils, pre- and post-bronchodilator spirometry, inhaled corticosteroid usage as well as standard demographic indices during stable scheduled follow-up visits. Linear mixed effects models were used to investigate the effect of log sputum eosinophils as a time varying covariate on decline of post bronchodilator $\mathrm{FEV}_{1}$. Models were iteratively compared and refined using standard information criteria. Other fixed effects in the final model were, time and the interaction terms for time * log sputum eosinophils and time *daily dose of inhaled corticosteroids and pack years smoked. Individual variations in the slopes and intercepts of time and time*log sputum eosinophils were considered by adding them iteratively as random effects. A first-order autoregressive correlation structure was used to model covariance of random effects.

Results 92 patients, $46 \%$ male with severe asthma were identified from a registry cohort of 686 between 2000 and 2009. The mean (sem) age was 54(12.9) years and age of onset 23 (2.1) years. The mean (range) duration of follow-up and number of visits were 6 years (4.6-10.5), 2.7/year. We found a significant interaction between sputum eosinophils, time and post bronchodilator $\mathrm{FEV}_{1}$. Indicating a net decline $(95 \% \mathrm{CI})$ of $-16.8 \mathrm{mls}(25.8-7.8 \mathrm{mls})$ /annum/log unit increase in sputum eosinophils ( $F(1,43.4)$; $\mathrm{p}<0.0001)$. In contrast there was a net decline $95 \% \mathrm{CI}$ of $-0.015 \mathrm{mls}$ $(0.029$ to $0.0014 \mathrm{mls}) / \mathrm{annum} / \mathrm{mcg}$ of inhaled beclamethasonediproprionate daily $(\mathrm{F}(1,726) ; \mathrm{p}=0.031)$.

Conclusion Eosinophilic airway inflammation is associated with a significant decline in $\mathrm{FEV}_{1}$ in severe asthma.

\section{S134 WITHDRAWN}

\section{S135 CAN YOUR MOBILE PHONE IMPROVE YOUR ASTHMA?}

doi:10.1136/thx.2010.150946.36

${ }^{1} \mathrm{D}$ Ryan, ${ }^{2} \mathrm{H}$ Pinnock, ${ }^{3} \mathrm{~L}$ Tarassenko, ${ }^{1} \mathrm{~A}$ Lee, ${ }^{2} \mathrm{~A}$ Sheikh, ${ }^{1} \mathrm{D}$ Price. ${ }^{1}$ Centre of Academic Primary Care, University of Aberdeen, Aberdeen, UK; ${ }^{2}$ Allergy and Respiratory Research Group, Centre for Population Health Sciences: GP Section, Edinburgh, UK; ${ }^{3}$ Department of Electrical Engineering, University of Oxford, Oxford, UK

Background It is recognised that some $45 \%$ of the population exhibit poor asthma control. Over $90 \%$ of the population possess a mobile phone ( $>70 \%$ over 60 years of age). Mobile technology potentially
Abstract S135 Table 1

\begin{tabular}{llll}
\hline AC06 score & Group 1(n=110) & Group 2(n=99) & $\begin{array}{l}\text { Between } \\
\text { group p value }\end{array}$ \\
\hline Baseline, median (IOR) & $2.17(1.67-2.67)$ & $2.33(1.67-2.67)$ & 0.441 \\
6 months, median (IOR) & $1.25(0.67-1.83)$ & $1.17(0.67-1.83)$ & 0.995 \\
Median difference (IQR) & $0.83(0.17-1.50)$ & $0.83(0.33-1.33)$ & 0.586 \\
Within group p value (Wilcoxon) & $<0.001$ & $<0.001$ & \\
& & & \\
Mean difference (95\% CI) & $0.84(0.67$ to 1.02) & $0.94(0.77$ to 1.11) & 0.434 \\
Within group $p$ value (t-test) & $<0.001$ & $<0.001$ & \\
MID*, n (\%) & & & 0.652 \\
Improvement = MID & $74(67.3)$ & $73(73.7)$ & \\
Improvement < MID & $20(18.2)$ & $16(16.2)$ & \\
Deterioration < MID & $6(5.5)$ & $5(5.5)$ & $5(5.1)$ \\
Deterioration = MID & $10(9.1)$ & & \\
\hline
\end{tabular}

addresses the barriers of low expectations and poor concordance which are factors in poor asthma control.

Hypothesis Using mobile phone recording of symptoms, lung function and medication use with instant feedback of asthma control, would improve control compared to using paper diaries.

Trial design A 6-month researcher-blinded randomised controlled trial

Setting UK primary care

Method Using central randomisation, we allocated patients $\geq 12$ years of age with poorly controlled asthma (ACQ $>1.5)$ to either mobile phone or paper-based monitoring. Clinical care was provided by practice asthma nurses in accordance with SIGN/BTS guidelines. Patients were reviewed monthly until control was achieved. A researcher, blinded to allocation assessed outcomes at $3 \mathrm{~m}$ and $6 \mathrm{~m}$. Primary outcome measure: change in Asthma Control Questionnaire score between baseline and 6 months.

Results We randomised 288 patients from 32 practices (209 completed). Baseline characteristics of both groups were similar. Intention to treat analysis, before breaking the randomisation code, showed that control in both groups improved significantly and to a similar extent. ACQ: Group $1(\mathrm{n}=110)$ Baseline 2.17, $6 \mathrm{~m}$ 1.25: Group 2 ( $\mathrm{n}=99)$ Baseline 2.33, 6m 1.17. Mean (95\% CI) improvement in ACQ: Group $1(n=110) 0.84(0.67,1.02)$, Group $2(n=99)$ $0.94(0.77,1.11)$ both $p<0.001$. Between group $p=0.434$ ns. Approximately $70 \%$ in each group improved by $\geq 0.5$ (minimal clinically important difference).

Conclusion Both groups demonstrated significant improvement in asthma control from baseline. Use of mobile phone technology provided no additional benefit over paper diaries.

\section{S136 FUNGAL SPUTUM CULTURE IN PATIENTS WITH SEVERE ASTHMA IS ASSOCIATED WITH A REDUCED POST BRONCHODILATOR FEV1}

doi:10.1136/thx.2010.150946.37

J Agbetile, A Fairs, M Bourne, B Hargadon, K Mutalithas, W Monteiro, R Edwards, J Morley, D Desai, C E Brightling, P H Bradding, R H Green, I D Pavord, A J Wardlaw, C H Pashley. Department of Infection, Immunity and Inflammation, Leicester. University \& Institute for Lung Health, Glenfield Hospital, Leicester, UK

Introduction and objectives IgE sensitisation to fungal allergens is common in severe asthma, but the clinical relevance of this, and the relationship to airway colonisation with fungi, is not known. Many of the fungi that can grow at body temperature are filamentous moulds from the genera Aspergillus and Pencillium. We report here the 